\title{
Muséologies
}

Les cahiers d'études supérieures

muséologies

\section{Entretien avec Monique Camirand}

\section{Charlène Bélanger}

Volume 6, numéro 2, 2013

La cybermuséologie

URI : https://id.erudit.org/iderudit/1018933ar

DOI : https://doi.org/10.7202/1018933ar

Aller au sommaire du numéro

Éditeur(s)

Association Québécoise de Promotion des Recherches Étudiantes en

Muséologie (AQPREM)

ISSN

1718-5181 (imprimé)

1929-7815 (numérique)

Découvrir la revue

Citer ce document

Bélanger, C. (2013). Entretien avec Monique Camirand. Muséologies, 6(2),

105-114. https://doi.org/10.7202/1018933ar

Tous droits réservés (C Association Québécoise de Promotion des Recherches Étudiantes en Muséologie (AQPREM), 2013
Ce document est protégé par la loi sur le droit d'auteur. L’utilisation des services d'Érudit (y compris la reproduction) est assujettie à sa politique d'utilisation que vous pouvez consulter en ligne.

https://apropos.erudit.org/fr/usagers/politique-dutilisation/ 
Dialogue deux

\section{Entretien avec Monique Camirand}

Charlène Bélanger 
Monique Camirand œuvre dans le monde de l'animation et de l'éducation depuis plus de trente ans. Pour être mieux outillée dans son travail, elle a complété deux baccalauréats - animation et recherche culturelle, et enseignement de l'art dramatique - ainsi qu'une maîtrise en sciences de l'éducation. Pour sa compagnie Paroles en Jeu, elle s'est spécialisée dans la conception d'expositions, la scénarisation de programmes éducatifs et la formation d'animateurs et de comédiens en contexte d'exposition et d'interprétation. De 2000 à 2012, elle a travaillé à titre de conseillère en éducation au Centre des sciences de Montréal et depuis peu elle a repris sa vie de consultante en éducation muséale. 
Depuis quelques années, nous avons vu apparaître la virtualité dans les musées et les centres de sciences. Par exemple, les collections de nombreux musées sont numérisées ou des audioguides ont été développés sous forme d'application iPhone. Pourriez-vous nous exposer quels ont été vos premiers contacts avec les technologies numériques dans votre pratique professionnelle?

Monique Camirand Dans les musées de science et technologie, les applications multimédias, les bornes interactives, les ordinateurs sont présents depuis déjà plusieurs années. Je me souviens de ma première expérience en muséologie, sous la direction de $\mathrm{M}^{\text {me }}$ Claude Benoît. C'était en 1984 à Québec dans le cadre des Grands Voiliers. Nous avions reçu le mandat de concevoir des expositions sur les thèmes du fleuve Saint-Laurent, des voiliers, de la navigation. Ce fut notre première école pour explorer différentes approches centrées sur une participation active des visiteurs dans leur processus de découverte et d'apprentissage. L'animation, les interactifs mécaniques et l'utilisation de différents moyens technologiques étaient au cœur de l'expérience. Puis, de 1988 à 1995 , toujours sous la direction de $\mathrm{M}^{\text {me }}$ Benoît, nous avons poursuivi notre exploration dans le cadre d'Expotech, des expositions à caractère scientifique et technique présentées chaque été dans le Vieux-Port de Montréal. Ce lieu est devenu par la suite le Centre des sciences de Montréal (en mai 2000). Dans ce type d'exposition, où il y avait peu de collections mais plutôt des espaces de démonstration et d'expérimentation, nous avions la possibilité d'observer et d'échanger avec les visiteurs pour constater que la plupart appréciaient vivre une expérience diversifiée pour apprendre.

Depuis quelques années, sur le plan de la médiation, nous assistons à un tournant majeur dans plusieurs musées de disciplines différentes. Un exemple d'une technologie qui vient de dépasser de nouvelles frontières est le spectacle Terra Mutantès du Musée de la nature et des sciences de Sherbrooke. Une aventure multimédia et multisensorielle: «Projetezvous au cœur de la formation des Appalaches et traversez des champs de lave... Vous ressentirez la séparation des continents et la déglaciation... La terre tremblera, vous aurez chaud, vous aurez froid... Vous ne verrez plus jamais le paysage des Cantons-de-l'Est du même œil! » Juste au toucher, le visiteur vit une expérience marquante qui s'inscrira probablement dans sa mémoire pour longtemps. C'est incroyable d'arriver à un tel niveau dans la transmission des contenus! Mais à quel coût! Nous constatons que la technologie a évolué très rapidement au cours des trente dernières années. Partant des postes informatiques, des applications multimédias, des bornes interactives, nous sommes passés à la tablette, au téléphone intelligent et, aujourd'hui, avec des exemples comme celui du musée de Sherbrooke, nous passons à un autre niveau: celui de l'apprentissage basé sur des sensations physiques intégrées aux nouvelles technologies.

Pourriez-vous nous parler d'un cas où vous considérez que l'introduction des nouvelles technologies dans les pratiques muséales a été un succès? 
M.C. Le meilleur exemple de réussite est à mon avis l'exposition Star Wars, présentée à l'été 2011 au Centre des sciences de Montréal. Je dois avouer qu'au début j'étais un peu sceptique par rapport à cette exposition. Je craignais l'éparpillement vers de nombreux objectifs: présenter une vaste collection de costumes, d'accessoires, de maquettes tirés des archives de Lucasfilm, présenter des extraits de films mettant en vedette les principaux personnages, donner des informations scientifiques sur les divers facteurs qui contribuent à façonner l'identité, et, en parallèle, engager le visiteur dans une quête sur sa propre identité en relation avec les héros de Star Wars. Tant de composantes auraient pu provoquer de la confusion ou une surcharge informationnelle du côté du visiteur. Au contraire, une intégration en parfaite harmonie.

À l'entrée de l'exposition, chaque visiteur recevait un capteur installé sur un cordon qu'il mettait à son cou, une oreillette, ainsi qu'un bracelet, pour enregistrer les réponses de sa quête. Dans chaque zone de l'exposition, le visiteur pouvait admirer les objets de la collection et faire des liens avec l'extrait de film présenté sur écran. Pour la quête, afin de rejoindre un plus grand nombre de visiteurs à la fois, il y avait plusieurs postes informatiques où l'on posait la même question. Le visiteur devait alors faire son choix de réponse sur l'écran tactile et celle-ci était enregistrée automatiquement sur son bracelet. Au bout du parcours de dix stations, le visiteur découvrait son identité par analogie avec l'un des personnages des films. Cette exposition fut une belle réussite! Durant la visite, les visiteurs pouvaient interagir avec les bornes informatiques parce qu'ils avaient les mains libres et échanger entre eux parce qu'ils avaient une oreille accessible. Ils pouvaient donc partager tout au long du parcours. Ils échangeaient des souvenirs, des impressions, des questionnements sur les facteurs d'identité. Même les plus jeunes étaient fascinés par les objets de la collection, par les extraits de films, et prenaient plaisir à réfléchir avec leurs parents et à répondre aux questions d'identité avec le bracelet. C'est ce que j'appelle un "Tintin », soit une très belle intégration pour les jeunes de 7 à 99 ans. Globalement, j'ai rarement vu une exposition qui arrivait à toucher autant tous les groupes d'âges.

Autre fait intéressant: à la fin de son parcours, le visiteur pouvait s'envoyer le résultat de sa quête par Internet et poursuivre son expérience à la maison en recevant un résultat plus détaillé de son profil. Cette approche permettait ainsi à une famille ou à un groupe d'amis de continuer à partager et à échanger sur leur profil identitaire en lien avec les personnages de Star Wars, même après leur retour à la maison.

\section{Pourriez-vous maintenant nous parler d'un cas où vous considé- rez que cela a plutôt été un échec?}

M.C. Oui, j'en connais un. Je pense ici à l'exposition Imagine du Centre des sciences de Montréal, ouverte de 2007 à 2012. Le concept de cette exposition était très intéressant. Nous avions l'impression d'entrer à l'intérieur d'un cerveau et, avec l'apport des nouvelles technologies, nous rencontrions virtuellement des créateurs et des inventeurs projetés sur des rideaux de vapeur d'eau. En nous approchant, nous pouvions les entendre raconter leur processus de création. Très technologique, 
mais très problématique aussi! La vapeur d'eau créait beaucoup d'humidité dans toute la zone. Le fonctionnement des pièces électroniques était touché et le système tombait régulièrement en panne. Cette technologie n'était pas d'entretien facile, mais elle était impressionnante lorsqu'elle fonctionnait.

Les écrans tactiles présentés dans cette exposition posaient également problème. Les visiteurs avaient de la difficulté à comprendre ce qu'il fallait faire. Même après l'ajout de l'illustration d'une main sur l'écran, il était difficile de savoir qu'il fallait d'abord toucher l'écran, puis glisser la main pour accéder à un contenu spécifique. Il n'y avait pas de consignes écrites pour guider le visiteur et c'était contre-intuitif. On se trouvait donc devant un problème de médiation. Malgré ces difficultés, il faut reconnaître que les concepteurs de l'exposition avaient eu le courage d'explorer une nouvelle avenue et on peut imaginer qu'un jour prochain les visiteurs sauront comment procéder pour vivre ce type d'expérience inusitée.

\section{Dans ce type d'exposition, on peut facilement imaginer que l'arrivée d'un groupe de trente élèves aux bornes informatiques peut compliquer le parcours de visite. Selon votre expérience, le public scolaire est-il pris en considération lors de la concep- tion du contenu numérique dans les expositions?}

M.C. Je ne peux pas parler pour toutes les institutions, mais au cours de mon expérience professionnelle, et selon ce que j'ai observé dans les expositions, je pense que les concepteurs des nouvelles expositions tiennent compte des groupes scolaires, car ils constituent aujourd'hui une grande partie de la clientèle d'un musée ou d'un centre d'interprétation. Si l'on revient à l'exemple de l'exposition Star Wars, chaque zone était autonome dans son message. Ce n'était pas un parcours linéaire. Nous pouvions donc faire le parcours dans le désordre, sans rien perdre au niveau des contenus ni de la quête. L'accueil des groupes scolaires est plus compliqué dans un parcours linéaire comprenant une série d'étapes à franchir dans l'ordre. Les concepteurs doivent donc, dès le début, réfléchir à l'expérience de visite, au parcours et à la gestion des groupes d'élèves de cycles différents. Par ailleurs, selon l'âge des jeunes, la présence d'un guide pour les encadrer est souvent requise. Contrairement à la visite guidée traditionnelle, il est possible d'avoir une approche à deux niveaux. Par exemple, le guide peut introduire le thème et les messages de chaque zone et, tout en demeurant disponible pour échanger et répondre aux questions, il laisse aux élèves le temps et le plaisir d'explorer, de manipuler, d'interagir avec les moyens technologiques pour ensuite conclure avec eux sur la zone et poursuivre la visite. Cette approche répond aux besoins des jeunes, qui demandent à vivre des expériences par eux-mêmes tout en étant encadrés. De plus en plus, les expositions sont conçues selon une approche non linéaire où chaque zone est autonome, où il y a plus d'un poste informatique, entre autres pour permettre une meilleure gestion du flux de visiteurs. Le problème ne se présente pas uniquement avec les groupes scolaires, on le voit aussi avec des groupes de touristes, ou lors d'une exposition très populaire où les visiteurs arrivent en grand nombre dès l'ouverture du musée. 


\section{Dans votre propre pratique professionnelle en éducation muséale, avez-vous eu l'occasion de développer des programmes éducatifs utilisant les technologies numériques?}

M.C. Oui, mais une seule fois, dans le cadre d'un programme intitulé Robot... clic! développé en 2000 pour le Centre des sciences de Montréal, à l'intention des élèves du troisième cycle du primaire. L'objectif visé était de permettre aux élèves d'apprendre à programmer et à faire fonctionner un robot de la première génération. C'était il y a treize ans. À ce moment-là, les enfants avaient un rapport très différent avec la technologie. Dès leur arrivée dans l'atelier de robotique, les jeunes étaient curieux et intrigués par la présence de plusieurs ordinateurs et différents montages construits en blocs Lego. Contrairement à certains logiciels instables que nous rencontrons parfois, celui-là était très bien fait. Sa force était la simplicité, dans l'utilisation et l'apprentissage, et cela demeure l'un de mes principes encore aujourd'hui : il est important de permettre aux jeunes de se centrer sur une démarche d'apprentissage spécifique qui mène à un résultat concret dans un laps de temps défini. Il faut toujours garder en tête que la participation à un atelier comme celui-là dure en moyenne soixante minutes. Cette réalité touche la plupart des institutions muséales. Nous sommes limités dans le temps pour faire vivre aux visiteurs, jeunes ou adultes, une expérience marquante qui permettra à chacun d'apprendre sur un thème spécifique. En une heure, il faut donc rester concentré sur un ou deux objectifs, tout au plus, et favoriser une participation active de chacun.

Ce projet fut une belle réussite, car, à l'époque, les enfants ne pouvaient vivre ce type d'expérience à la maison. Programmer comme des grands et voir immédiatement le résultat concret en observant l'automate en mouvement (une porte de garage qui s'ouvre, un manège qui tourne, etc.). Ce programme éducatif a connu un grand succès tant auprès des élèves que des enseignants. Toutefois, au fil des ans, les jeux vidéo devenaient de plus en plus populaires chez les jeunes, même en bas âge. Notre programme atteignait toujours ses objectifs, mais nous percevions une différence au niveau de l'émerveillement des jeunes. Comme la motivation et la curiosité sont des facteurs importants de l'apprentissage, nous avons décidé de mettre fin à ce programme en 2007.

\section{L'utilisation des technologies numériques dans les actions de médiation génère-t-elle des problèmes particuliers? Avez-vous des exemples en tête?}

M.C. Oui. Il peut y en avoir plusieurs, mais je préfère plutôt énoncer des principes à partir d'un exemple précis que l'on peut voir dans divers musées ou centres d'interprétation. Je me réfère à l'exemple d'un module interactif dans lequel se trouvent un dispositif multimédia, un jeu-questionnaire avec choix de réponses, une durée limitée dans le temps pour répondre et un pointage de performance à la fin. C'est, selon moi, trop de composantes dans un processus de médiation et d'apprentissage. Ce genre d'activité est très populaire auprès des jeunes, car l'approche ressemble à celle d'un jeu vidéo. On lance un défi aux visiteurs: "Vous avez tant de minutes pour répondre et battre le record.» C'est l'esprit de compétition qui est sollicité ici, et ce, au détriment d'autres 
activités importantes. Par exemple, ils ne prennent pas le temps de lire, de vraiment comprendre les contenus même s'ils sont courts et bien écrits: Les liens entre chaque composante sont difficiles à faire car le temps est limité. Le but, c'est le pointage. À la suite de mes observations en salle, j'ai demandé à quelques jeunes ce qu'ils aimaient de ce type d'activité. Tout de suite, j'ai obtenu des réponses comme: "J'ai compris comment répondre pour faire plus de points! » ou «J'ai battu mon père! » Aucune réponse sur le message principal, pourtant très intéressant. Du côté des adultes, j'ai observé qu'ils ne se rendaient pas au bout de l'activité. Certains m'ont dit manquer de temps pour lire les questions et réfléchir, ne serait-ce que quelques secondes, aux choix de réponses, car rapidement le système les amenait à une autre étape. Cette situation nuit à la concentration, peut provoquer un certain stress et amener les visiteurs à décrocher avant d'avoir terminé. Je pense qu'il faut faire attention à vouloir trop ressembler aux jeux vidéo, ou à trop vouloir aller dans la performance. Lancer un défi, c'est bien, mais il ne faut pas viser trop d'actions en même temps. J'ai énormément de difficultés avec cette approche multitâche qui s'éloigne des principes fondamentaux en éducation. Une compétition saine peut être un moteur pour apprendre, mais non une finalité.

\section{À propos du multitâche, est-ce que vous croyez que les visiteurs ont les compétences nécessaires à la gestion des nombreuses informations qui leur arrivent simultanément selon différentes modalités, comme la vidéo, l'objet, le texte, l'audio?}

M.C. Je pense que les jeunes sont déjà très habitués au multitâche. Ils sont de plus en plus nombreux à faire plusieurs activités en même temps... Ils écoutent la télé ou sont branchés sur leur musique, ils ont l'ordinateur sur eux pour être en contact sur Facebook, sans oublier le téléphone cellulaire à proximité au cas où... Tout ça en même temps! Je pense que les jeunes ont la compétence requise pour utiliser les outils et gérer les multiples informations dans une exposition. Et quand je parle des jeunes, je parle des jeunes jusque dans la vingtaine et même la trentaine. C'est la génération qui a grandi avec ces appareils. Ils visitent l'exposition avec un iPod et des écouteurs sur les oreilles. J'en vois qui répondent à des SMS [textos] ou qui visiten leur page Facebook. En même temps qu'ils visitent l'exposition, ils continuent donc d'être ailleurs, dans leur vie personnelle, professionnelle, dans leur réseau social virtuel. Et, dans ce contexte, que retiennent-ils des messages principaux de l'exposition et de l'expérience proposée?

En somme, je pense que les jeunes ont la compétence nécessaire, mais je ne connais pas l'impact sur l'acquisition de nouvelles connaissances. Quant aux autres catégories de visiteurs, même les plus âgés, plusieurs apprécient de plus en plus la visite avec un appareil de style iPod. Contrairement à la visite guidée qui les oblige à écouter un guide qui impose un parcours et un rythme, ils choisissent le iPod pour être en contrôle de leur visite. Par contre, j'ai pu observer qu'il arrive fréquemment que les visiteurs circulent chacun de leur côté car l'appareil ne 
favorise pas l'échange entre eux. L'expérience de visite est donc moins sociale. Je pense cependant que les gens sont satisfaits en bout de ligne, parce qu'ils ont visité à leur rythme, ils ont vu ce qu'ils voulaient voir.

Mais il semble que cela ne soit pas unique à l'appareil iPod, les audioguides classiques ne réduisent-ils pas aussi la portée sociale de la visite?

M.C. Oui, en effet, c'est le même constat pour l'audioguide. Cette réalité n'a pas changé, c'est le moyen qui a changé. Comme je l'ai exposé précédemment, je pense qu'il est possible de développer des concepts technologiques qui n'aient pas cet effet d'isolement. La preuve: dans l'exposition Star Wars, grâce au bracelet mains libres et à l'oreillette unique, les interactions sociales étaient favorisées durant toute la durée de la visite et elles se poursuivaient ensuite jusqu'à la maison!

Les musées offrent aujourd'hui la possibilité de louer un appareil de type iPod ou autre, ou d'utiliser son appareil personnel dans l'espace du musée. Croyez-vous que l'expérience de visite offerte dans ces deux cas soit comparable?

M.C. La personne qui visite avec son propre appareil a l'avantage de savoir l'utiliser efficacement. Par contre, comme je le disais un peu plus tôt, elle peut aussi décider d'aller naviguer sur le Web, de visiter sa page Facebook ou autre réseau social. Dans le métro ou dans la rue, il est courant de voir des passants avec leur appareil à la main, toujours en contact avec leur propre monde virtuel. C'est une habitude qui s'est développée et ça ne s'arrêtera pas nécessairement à l'entrée du musée. Tandis que le visiteur qui loue ou emprunte un appareil au musée n'aura pas d'accès à Internet. Il restera donc centré sur l'exposition et ses contenus.

\section{L'introduction de nouvelles technologies au musée exclut-elle ou éloigne-t-elle certains publics de l'institution?}

M.C. Non, plus maintenant. Il y a dix ans, on sentait une résistance face à l'appareil, surtout chez les visiteurs les plus âgés. Aujourd'hui, il y a un intérêt marqué pour la technologie parmi toutes les tranches d'âges. Mais il y a quand même des personnes, peu importe l'âge, qui n'aiment pas ou sont mal à l'aise avec la technologie, les ordinateurs. Et ces personnes-là ne sont pas exclues pour autant du musée. Elles peuvent encore choisir d'autres modalités de visite et des activités de médiation qui leur conviennent davantage. La visite guidée est encore très populaire, car plusieurs souhaitent avoir un contact humain pour en savoir davantage sur une thématique et pouvoir échanger et questionner pour apprendre.

Selon vous, est-ce que la volonté d'introduire les nouvelles technologies induit des contraintes, des difficultés nouvelles dans les pratiques muséales, notamment au niveau des coûts ou de l'organisation du travail?

M.C. Au niveau des coûts, cela dépend de la complexité de l'appareil ou de la technologie, bien sûr. Lorsque c'est une borne interactive simple, c'est moins élevé. Lorsque cela devient multimodal (images, textes, vidéos), on peut se retrouver avec des coûts faramineux. Si 
l'on prend l'exemple que j'ai évoqué au tout début de cette entrevue, la table interactive et multisensorielle Terra Mutantès présentée au Musée de la nature et des sciences de Sherbrooke, la conception aura nécessité près de cinq années de travail et un budget de 720000 \$. C'est très innovant et impressionnant, mais les moyens mobilisés sont énormes!

Par ailleurs, dans le développement des nouvelles technologies, les musées doivent aujourd'hui travailler avec des équipes multidisciplinaires composées de designers, de programmeurs, d'ingénieurs, de scénaristes, de techniciens et parfois d'éducateurs ou de muséologues. Ce sont fréquemment des jeunes adultes « accros » à la technologie qui ont appris très tôt à travailler en coopération. Ils se complètent et sont souvent très créatifs. C'est vraiment stimulant, mais cela requiert une nouvelle répartition du travail.

Le côté un peu moins réjouissant, c'est peut-être la fragilité des appareils conçus à partir des nouvelles technologies, surtout s'ils sont manipulés par des milliers de visiteurs au fil des mois et des années. Cette réalité requiert un personnel qualifié, idéalement sur place et prêt à résoudre rapidement les problèmes. De nos jours, certains musées inscrivent dans leurs devis d'appels d'offres une clause qui demande à l'équipe de conception de garantir le bon fonctionnement de l'outil pendant les premiers mois, voire une année. Ils sont donc responsables du suivi et des réparations dans le cadre de leur planification budgétaire. Je trouve cette clause importante, car elle oblige l'équipe de conception à beaucoup plus de vigilance quant à la qualité et la durabilité du produit technologique.

Globalement, il est difficile d'évaluer si l'arrivée des technologies facilite ou alourdit la tâche des musées. Cela dépend des situations. Par exemple, programmer une application pour iPod ou iPhone peut exiger un travail monumental. Ce n'est plus simplement un audioguide avec une narration. Nous avons désormais accès à bien davantage: on peut, en plus de la narration, voir des photos, des extraits vidéo associés au contexte thématique, obtenir de l'information complémentaire et même du 3D. C'est incroyable de voir toutes les possibilités reliées à cette nouvelle technologie. Mais la somme de travail que cela peut exiger de l'équipe du musée est énorme, sans compter les spécialistes externes qu'il faut engager. À mon avis, le développement d'applications technologiques pour des expositions permanentes est une avenue intéressante. Or, dans le cas d'expositions temporaires, cela demeure un peu risqué, puisque cela demande beaucoup de temps, d'investissement et d'énergie de la part des équipes de travail. En outre, la compétition est très féroce autour des expositions temporaires et tout le monde veut avoir son iPod, sa tablette, son outil technologique, car c'est "vendeur " auprès du visiteur. Alors peut-être est-ce devenu un incontournable! Par contre, si l'exposition peut voyager et être offerte dans d'autres villes, provinces ou pays, c'est différent. Cela permet de rentabiliser l'outil à long terme.

Au cours de cette entrevue, vous nous avez rapporté certains constats de succès ou d'échec d'innovations technologiques dans les musées. Dans votre pratique, avez-vous réalisé des études 


\section{de publics et des évaluations des nouvelles technologies? Et avez-vous accès aux résultats de recherches qui auraient été réalisées par d'autres musées ou institutions?}

M.C. Les exemples dont je vous ai parlé aujourd'hui étaient fondés sur des observations à la suite de visites dans divers musées ou d'évaluations formelles réalisées par le Centre des sciences de Montréal, où j'ai travaillé pendant plusieurs années. Les éducateurs, les animateurs ou les guides côtoient tous les jours le public, observent les visiteurs et recueillent leurs commentaires. Ces commentaires sont très importants pour nous aider à évaluer les expositions et les programmes éducatifs. Pour ma part, en collaboration avec Marie-Thérèse Bournival, nous avons réalisé une étude sur l'animation des publics dans un contexte d'exposition. C'était en 1989, à l'époque où les nouvelles technologies n'étaient pas très présentes dans les institutions muséales. À ma connaissance, la seule professionnelle spécialisée en évaluation à avoir réalisé des études sur ce sujet est Lucie Daigneault du Musée de la civilisation à Québec. C'est un thème très intéressant et le temps nous manque sur le terrain pour faire ce type de recherche. Heureusement, il y a de plus en plus d'ouverture du côté des universités pour travailler en collaboration avec les musées et permettre un meilleur partage des connaissances. Toutefois, s'il existe des résultats de recherche sur les publics et les nouvelles technologies, et sur le multitâche dans un processus de médiation, je souhaiterais qu'ils soient diffusés sur une plate-forme commune, par exemple sur le site de la Société des musées québécois, pour que tous y aient accès. 